\title{
State of the art? The CAGE study L'avant-garde? L'étude CAGE
}

$I^{n}$ $\mathrm{n}$ the present issue of the Canadian Respiratory Journal, Bourbeau et al (1) publish results of a survey designed to assess the adequacy of chronic obstructive pulmonary disease (COPD) therapy in Quebec and Ontario. They recruited 161 primary care physicians in the two provinces, who in turn recruited 1090 patients with COPD. The patients were assessed by their physicians in terms of status and therapy. Patient demographics were noted, as was therapy, which was compared with recommended Canadian Thoracic Society (CTS) guidelines (2). In addition, nonpharmacological treatments in terms of smoking cessation, influenza vaccination and rehabilitation were assessed, as was the frequency of spirometry. Patients were categorized using the Medical Research Council (MRC) dyspnea scale, as outlined in the CTS guidelines.

The result was a snapshot of COPD evaluation and management, approximating practice in the real world, which is of considerable interest, because it has been shown that primary care physicians are the major caregivers for $80 \%$ of COPD patients (3). There were several important discrepancies between the guidelines and the real world. First, approximately $14 \%$ of the patients with (nominal) COPD were not dyspneic according to the MRC scale administered by their physicians. Probably in large part because of this, there was only 'fair' agreement between physicians' assessment of disease severity and that of the guidelines, with severity being overestimated both by the physicians and the patients themselves. It is likely, though not stated, that many of these patients were given drugs not recommended by the guidelines.

In symptomatic patients, drug therapy administered by the physicians was less discriminating than that recommended by the guidelines, resulting in patients with relatively mild COPD receiving more drugs, and patients with severe COPD receiving less therapy than recommended. This was particularly notable for inhaled corticosteroids, which were given to approximately $60 \%$ of patients irrespective of severity. This was not explained by potential confusion with asthma, because relatively few patients carried this label. Long-acting bronchodilators were given to approximately $40 \%$ of patients, again irrespective of severity. Only approximately $50 \%$ of the severely ill patients were given two long-acting bronchodilators in compliance with the guidelines.

Almost all of the patients received advice regarding smoking cessation - approximately $40 \%$ were still smoking - and the vast majority had annual flu vaccinations, both good things. Rehabilitation was used by only $9 \%$ of moderately or severely ill patients, reflecting the general lack of access to rehabilitation facilities. Slightly more than $50 \%$ of the patients had undergone spirometry at some point in their illness; this was more common in those with severe disease, but related only weakly to adherence to pharmacological guidelines.
Dans le présent numéro du Journal canadien de Dneumologie, Bourbeau et coll (1) publient les résultats d'une enquête conçue pour vérifier si le traitement de la maladie pulmonaire obstructive chronique (MPOC) se fait correctement au Québec et en Ontario. Les chercheurs ont recruté 161 omnipraticiens des deux provinces qui, à leur tour, ont inscrit 1090 patients atteints de MPOC. Les patients ont été évalués par leur médecin sur le plan de leur statut fonctionnel et de leur traitement. Leurs caractéristiques démographiques et leurs traitements ont été consignés, puis comparés aux lignes directrices recommandées par la Société canadienne de thoracologie (SCT) (2). On a en outre évalué les approches non pharmacologiques, notamment l'abandon du tabac, la vaccination antigrippale et la réadaptation, de même que la fréquence de la spirométrie. Les patients ont été classés en catégories selon l'échelle de mesure de la dyspnée du Conseil de recherche médicale (CRM) présentée dans les directives de la SCT.

L'enquête a permis de dresser un tableau de la pratique actuelle en matière d'évaluation et de prise en charge de la MPOC, ce qui est fort intéressant puisqu'il a été démontré que ce sont surtout les omnipraticiens qui soignent ce type de patients (80\%) (3). Plusieurs écarts importants ont été notés entre les lignes directrices et la pratique courante. Tout d'abord, environ $14 \%$ des patients porteurs d'un diagnostic de MPOC n'étaient pas dyspnéiques si l'on se fie au questionnaire du CRM administré par les médecins. Cela s'explique probablement en bonne partie du fait qu'il y avait une concordance seulement «passable » entre l'évaluation de la gravité de la maladie par les médecins et l'évaluation décrite dans les lignes directrices, la gravité ayant été surestimée, tant par les médecins que par les patients eux-mêmes. Bien que cela ne soit pas précisé, on peut présumer que bon nombre de ces patients ont donc reçu des médicaments non recommandés dans les lignes directrices.

Chez les patients symptomatiques, le traitement médicamenteux administré par les médecins était moins pointu que le traitement recommandé dans les lignes directrices. Ainsi, les patients atteints d'une MPOC relativement légère ont reçu plus de médicaments et les patients plus gravement atteints en ont reçu moins qu'il n'est recommandé. Ce phénomène était particulièrement observable dans le cas des corticostérö̈des par inhalation, qui ont été administrés à environ $60 \%$ des patients, peu importe la gravité de leur état. Cela n'a pas été attribué à une possible confusion avec l'asthme, puisqu'un nombre relativement faible de patients étaient porteurs de ce diagnostic. Les broncho-dilatateurs à longue action ont été administrés à environ $40 \%$ des patients, encore une fois peu importe la gravité de leur état. Seulement 50 \% environ des grands malades prenaient deux bronchodilatateurs à longue action, conformément aux directives.

Presque tous les patients ont été avisés de cesser de fumer environ $40 \%$ fumaient toujours - et la grande majorité recevait une vaccination antigrippale annuelle, deux mesures préconisées. La réadaptation a été utilisée chez $9 \%$ seulement des patients modérément ou gravement atteints, ce qui témoigne de la 
There were differences between physicians in Ontario and Quebec, in that the former used more long-acting bronchodilators and spirometry, and the latter were more likely to use rehabilitation services. These discrepancies almost certainly relate to access as opposed to philosophy.

What are we to make of this? Is the glass half full or half empty? A sobering thought is that the primary care physicians who formed the base of the study were self-selected and therefore more likely to be interested and informed than the community at large. Indeed, most had attended at least one COPD-related education session in the past year. Thus, things in the really real world are likely to be worse than portrayed by Bourbeau et al (1), something noted by the authors. A second thought might be that the guidelines are needlessly discriminating, classifying patients excessively. It is difficult for me to accept this. Patients who are short of breath only when hurrying are different from those who have trouble with virtually any activity, and I regard the use of the symptom-based MRC scale as one of the strengths of the CTS guidelines. It seems that the physicians participating in the Bourbeau study had a tendency to treat all patients with all drugs, except for the most severe cases. As Bourbeau et al (1) point out, undertreating severe patients is likely to be bad for their quality of life, and over-treating mild ones is expensive, although probably not dangerous.

I suppose that we should not be surprised by these findings by Bourbeau et al (1), who clearly regard the glass as half empty, and it is difficult to dispute their conclusion that we need to do better in making guideline-related therapy the norm and not the exception.

Nick R Anthonisen MD Editor-in-Chief, Canadian Respiratory Journal

\section{REFERENCES}

1. Bourbeau J, Sebaldt RJ, Day A, et al. Practice patterns in the management of chronic obstructive pulmonary disease in primary practice: The CAGE study. Can Respir J 2008;15:13-19.

2. O'Donnell DE, Aaron S, Bourbeau J, et al. Canadian Thoracic Society recommendations for management of chronic obstructive pulmonary disease - 2003. Can Respir J 2003;10(Suppl A):11A-65A.

3. Chapman KR, Bourbeau J, Rance L. The burden of COPD in Canada: Results from the Confronting COPD survey. Respir Med 2003;97(Suppl C):S23-31. difficulté d'accéder à ce type de service. Un peu plus de $50 \%$ des patients avaient subi une épreuve de spirométrie à un moment ou à l'autre au cours de leur maladie; cette mesure a été appliquée davantage chez les malades plus gravement atteints, mais elle était seulement en faible corrélation avec la fidélité aux directives pharmacologiques.

On a noté des différences entre les médecins de l'Ontario et ceux du Québec, les premiers utilisant davantage les bronchodilatateurs à longue action et la spirométrie et les seconds étant plus susceptibles de recourir aux services de réadaptation. Cette distinction est sûrement liée davantage à une question d'accessibilité qu'à une question de principe.

Quelles conclusions faut-il tirer de tout cela? Le verre est-il à moitié plein ou à moitié vide? Il faut retenir que les omnipraticiens qui formaient la base de l'étude s'y étaient portés volontaires et étaient donc plus susceptibles de s'y intéresser et de s'informer, comparativement à d'autres médecins. En effet, la plupart avaient participé à au moins un atelier de formation sur la MPOC au cours de l'année écoulée. Ainsi, comme le font remarquer les auteurs, dans la «vraie » vie, la situation est peutêtre moins réjouissante que dans l'étude de Bourbeau et coll (1). On peut également arguer que les directives sont trop pointues et classent les patients selon des critères trop stricts. J'ai du mal à accepter cette position. Les patients qui ne sont essoufflés que lorsqu'ils se hâtent sont différents des patients qui le sont au moindre effort et je considère l'utilisation de l'échelle du CRM établie selon les symptômes comme l'un des principaux atouts des directives de la SCT. Il semble que les médecins qui ont participé à l'étude de Bourbeau aient eu tendance à traiter tous les patients avec tous les médicaments, à l'exception des cas les plus graves. Comme le signalent Bourbeau et coll (1), chez les patients gravement atteints, un traitement insuffisant risque de nuire à la qualité de vie, tandis que dans les cas légers, un traitement trop énergique se révèle coûteux, quoique probablement sans danger.

Je suppose que nous ne devrions pas nous surprendre de ces observations de Bourbeau et coll (1) qui considèrent nettement le verre à moitié vide. Il est difficile de contester leur conclusion selon laquelle l'adhésion aux directives thérapeutiques devrait être la norme et non l'exception.

Nick R Anthonisen, MD Rédacteur en chef, Journal canadien de pneumologie

\section{RÉFÉRENCES}

1. Bourbeau J, Sebaldt RJ, Day A et coll. Practice patterns in the management of chronic obstructive pulmonary disease in primary practice: The CAGE study. Can Respir J 2008;15:13-19.

2. O'Donnell DE, Aaron S, Bourbeau J et coll. Canadian Thoracic Society recommendations for management of chronic obstructive pulmonary disease - 2003. Can Respir J 2003;10(Suppl A):11A-65A.

3. Chapman KR, Bourbeau J, Rance L. The burden of COPD in Canada: Results from the Confronting COPD survey. Respir Med 2003;97(Suppl C):S23-31. 


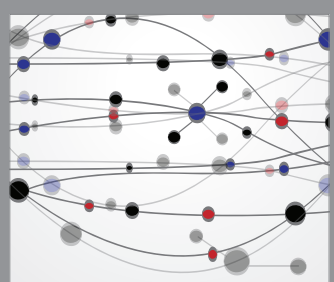

The Scientific World Journal
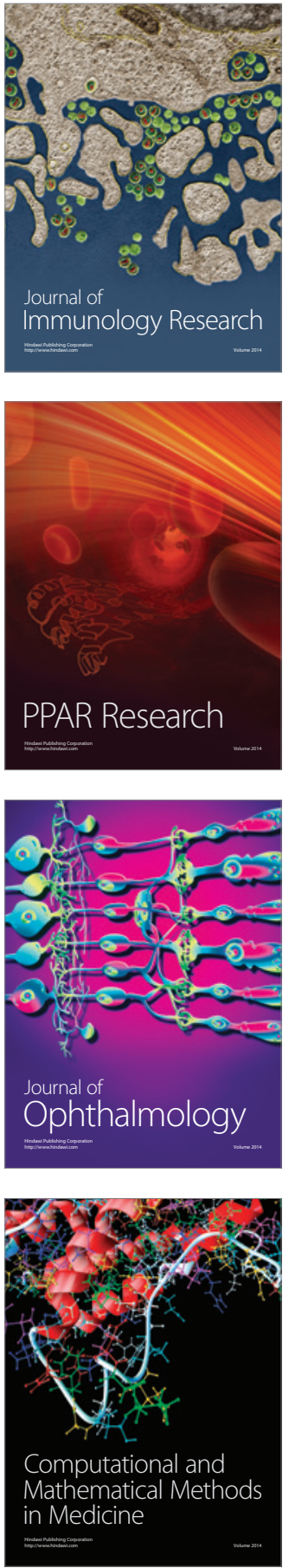

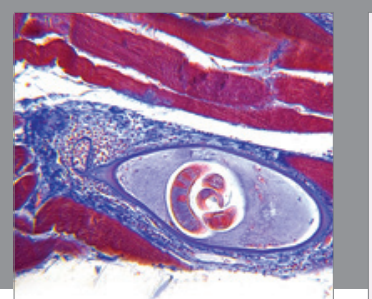

Gastroenterology Research and Practice

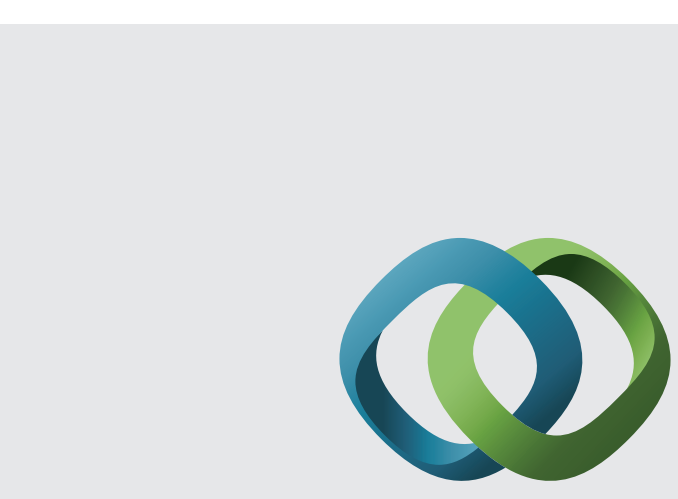

\section{Hindawi}

Submit your manuscripts at

http://www.hindawi.com
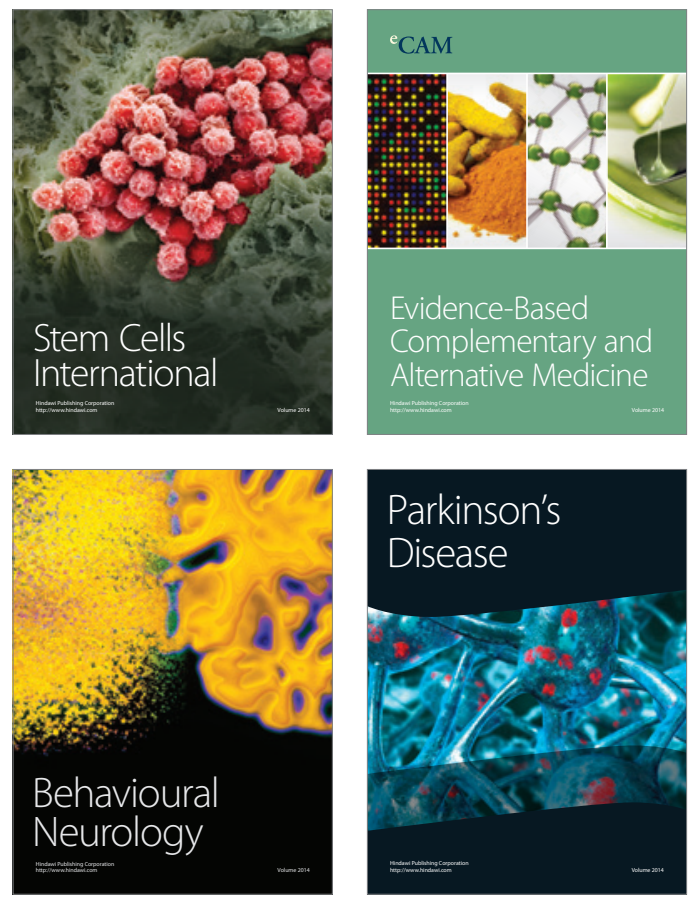
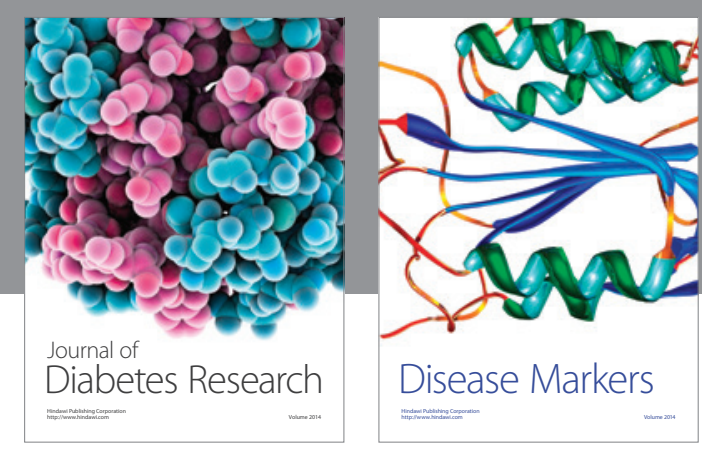

Disease Markers
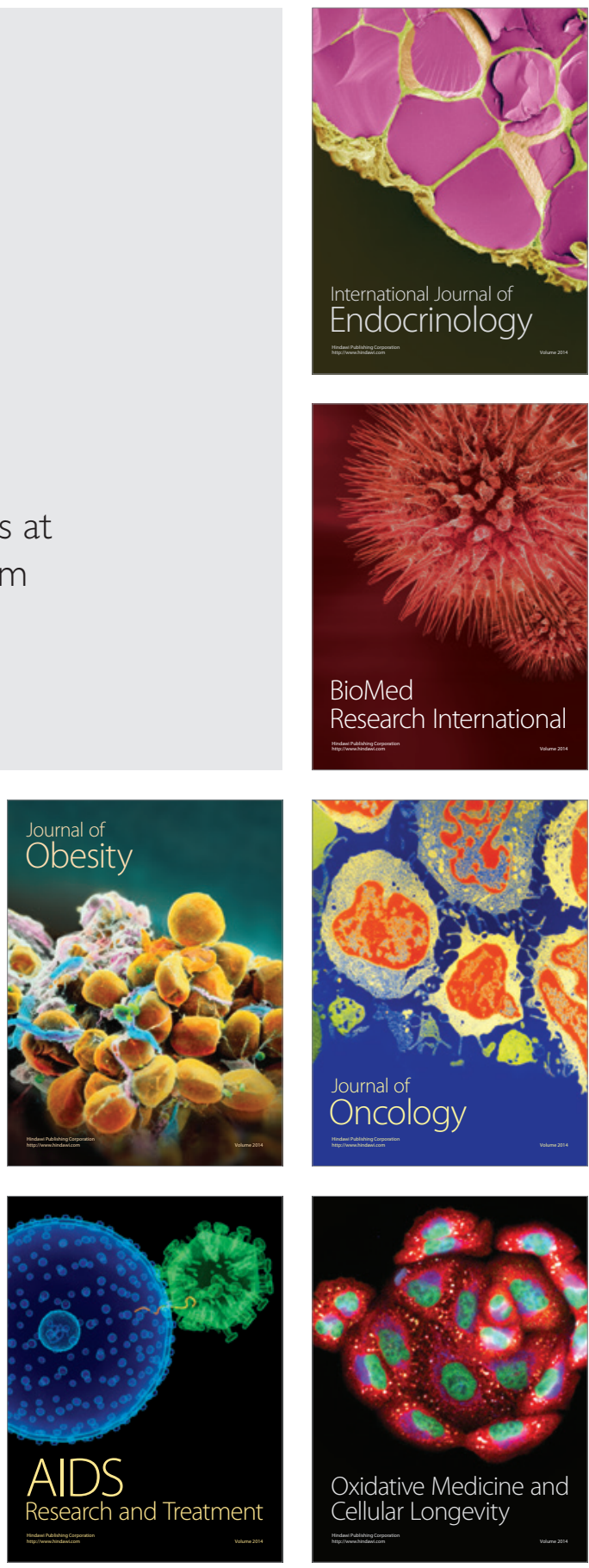